\title{
SÍNDROME DE EMBOLISMO GRASO
}

\author{
Carlos Alberto Navarro Lozano, MD.*, Juvenal Bayona Mora, MD.**
}

\section{Resumen}

Antecedentes: los pacientes con fracturas diafisiarias de huesos largos con hipovolemia e hipoxia presentan mayor riesgo del síndrome de embolismo graso (SEG). El manejo temprano con líquidos endovenosos y oxigenoterapia puede prevenirlo. La incidencia en el hospital de San José es $7 \%$.

Objetivo: establecer la frecuencia de SEG en los pacientes que acudieron entre febrero y diciembre de 2003, con fracturas diafisiarias de fémur y/o tibia, manejados desde el ingreso con líquidos endovenosos y oxígeno.

Diseño: estudio observacional, retrospectivo y descriptivo, tipo serie de casos.

Lugar: hospital de San José (nivel IV).

Resultados: de 59 pacientes, 35 (58\%) ingresaron a urgencias en los primeros 60 minutos después del trauma, 26 (44\%) presentaron fractura cerrada de tibia y 18 (30\%) fractura cerrada de fémur. Diez se consideraron polifracturados (17\%) y 3 (5\%) politraumatizados. 48 (81\%) sufrieron traumas de alta energía. En las primeras 24 horas se estabilizaron quirúrgicamente el $56 \%, 32$ de ellos con clavos endomedulares con una duración promedio de 122 minutos. La incidencia de fracturas aisladas de fémur o tibia fue de $\mathbf{2 . 0 4} \%$, de las cuales en politraumatizados fue de $10 \%$. Los dos pacientes ingresaron en la primera hora postrauma con fracturas diafisiarias cerradas de fémur, uno con fractura tibial contralateral abierta y se estabilizaron dos horas después de su ingreso con fijadores externos en 80 minutos promedio. Ningún paciente falleció.

Discusión: 1. El estudio no muestra influencia contundente de alguna de las variables manejadas, esto permite pensar que el SEG depende más de la respuesta propia del huésped a los ácidos grasos que de su origen. 2. La incidencia de SEG en este trabajo fue menor que la reportada en un estudio anterior en esta misma institución con igual población de pacientes, donde no se utilizó protocolo de líquidos endovenosos y oxígeno.

Palabras clave: Embolismo graso, fractura fémur, fractura tibia, prevención, líquidos parenterales, oxígeno.

\section{Introducción}

El síndrome de embolismo graso (SEG) es una complicación del trauma esquelético. Puede ser de origen traumático o no. El embolismo graso y el síndrome de embolismo son dos entidades diferentes. ${ }^{1}$ El primero se define como la presencia de glóbulos grasos en el parénquima pulmonar y la circulación periférica. ${ }^{2}$ Según Palmovic y Mc Carroll, en aproximadamente el 90\% de las fracturas de huesos largos ocurre embolismo graso., ${ }^{2,3}$ El SEG es la manifestación más severa del embolismo graso. La incidencia es del 0.5 a $3 \%$ en fracturas aisladas de huesos largos y de $10 \%$ en polifracturados . La mortalidad puede llegar a ser tan alta como del 10 al 20\%. ${ }^{4}$

\footnotetext{
* Ortopedista y traumátologo Hospital de San José. Clínica Fundadores. Instructor de Técnicas Quirúrgicas en Ortopedia, Facultad de Instrumentación, Fundación Universitaria de Ciencias de la Salud.

* Residente IV año Ortopedia y Traumatología Hospital de San José.

*** Este trabajo fue realizado bajo la tutoría del Dr. Guillermo Rueda, ortopedista y traumatólogo. Instructor Asociado Servicio de Ortopedia y Traumatología, Hospital de San José y del Dr. Carlos Mario Olarte, ortopedista y traumatólogo. Instructor Asociado, Hospital de San José.
}

En cuanto a su patofisiología hay dos teorías del efecto de los émbolos grasos. La teoría mecánica: el trauma produce ingreso de células grasas a la circulación venosa y por ende al pulmón, donde ocasionan oclusión de los vasos más pequeños, la cual aumenta por la adherencia de plaquetas y fibrina al glóbulo graso. ${ }^{1,2}$ La teoría bioquímica propone que una vez las células grasas ingresan a la circulación pulmonar, las lipasas pulmonares las hidrolizan convirtiendo estos émbolos neutros inactivos en ácidos grasos libres tóxicos que disparan la cascada inflamatoria, la cual a su vez lleva a daño endotelial, inactivación del surfactante pulmonar e incremento en la permeabilidad capilar.

Este último evento produce edema pulmonar intersticial y finalmente síndrome de dificultad respiratoria del adulto (SDRA). ${ }^{1,2,4-7}$ En la actualidad la teoría bioquímica tiene mayor aceptación en la explicación de la fisiopatología del SEG. Entre 30 y 50\% de los pacientes con fracturas de huesos largos presenta hipo- 
xemia en los gases arteriales, la cual es transitoria y se debe a la presencia de émbolos grasos subclínicos. ${ }^{1}$

Para que se presente el SEG debe haber factores predisponentes entre los cuales se han mencionado el choque y la hipovolemia, ${ }^{1,7}$ patologías que se presentan con frecuencia en el paciente politraumatizado y polifracturado.

Los gases arteriales son la ayuda diagnóstica más útil para la detección temprana del SEG. "Es el procedimiento diagnóstico esencial y debe ser realizado tempranamente después de la admisión y frecuentemente durante las siguientes 48 horas en todos los pacientes con trauma óseo significativo" según Peltier. ${ }^{8}$ Una $\mathrm{paO}_{2}$ menor de $60 \mathrm{~mm}$ de $\mathrm{Hg}$ después de trauma esquelético en un paciente que respira espontáneamente sin otra patología pulmonar, sugiere SEG ${ }^{1,5}$

El desarrollo del SEG puede prevenirse mediante inmovilización adecuada, transporte rápido, uso de oxigenoterapia en el período postlesión y estabilización temprana de las fracturas de huesos largos de las extremidades inferiores. Son las tres medidas importantes que deben tomarse en cuenta para reducir la incidencia de esta complicación. La evaluación de la presión arterial, los gases arteriales y en el paciente muy lesionado la presión en cuña pulmonar, son útiles para valorar el estado de los fluidos y la perfusión tisular. La oxigenoterapia con máscara previene la caída de la oxigenación arterial debido a fractura y parece tener valor en la prevención del SEG. ${ }^{9}$

Como ya se ha mencionado, uno de los factores que predisponen al desarrollo del SEG es la hipovolemia. Por lo tanto, se debe realizar una reanimación adecuada de estos pacientes en urgencias con líquidos intravenosos (cristaloides) en bolo de 2000 cc como lo recomienda el ATLS (Advance Trauma Life Support).

En el hospital de San José de Bogotá se recibe en el servicio de urgencias un número creciente de pacientes con fracturas diafisiarias de fémur y tibia, entre los cuales se ha visto el SEG. Han requerido manejo en unidad de cuidado intensivo (UCI), presentando una morbilidad elevada con altos costos y complicaciones que han llegado hasta la muerte. El manejo temprano de los pacientes con fracturas diafisiarias de fémur y/o tibia mediante líquidos endovenosos y oxígeno, permitiría disminuir la incidencia y mortalidad en el SEG.

\section{Materiales y métodos}

- Tipo de estudio: observacional retrospectivo, descriptivo, tipo serie de casos.

- Población y muestra: pacientes que ingresaron al servicio de urgencias del hospital de San José de Bogotá, con diagnóstico de fractura diafisiaria de fémur y/o tibia, entre febrero y diciembre 2003, con protocolo de manejo temprano con líquidos y oxígeno.

- Criterios de inclusión: pacientes mayores de 15 años y menores de 60 años; diagnóstico de fractura diafisiaria de fémur y/o tibia; período entre febrero de 2003 y diciembre de 2003 y aplicación de protocolo de manejo temprano con líquidos y oxígeno.

- Criterios de exclusión: fracturas patológicas diafisiarias de fémur y/o tibia, manejo quirúrgico previo en otra institución y error diagnóstico inicial en el servicio de urgencias del hospital de San José.

\section{Resultados}

Se revisaron 68 historias de pacientes con diagnóstico de fractura diafisiaria de fémur y/o tibia, de las cuales 59 cumplieron criterios de inclusión. Se excluyeron 9 pacientes a los cuales no se les realizó protocolo de manejo temprano con líquidos endovenosos y oxígeno por causas no aclaradas. Se encontró predominio del sexo masculino con 52 casos (88\%) . Las pacientes del sexo femenino fueron 7 (12\%) (Figura 1). El rango de edad entre los 25 y 35 años aportó 23 casos (40\%), seguido por el rango entre 14 y 24 años con 22 (37\%). El intervalo de tiempo entre la ocurrencia del trauma y el ingreso al servicio de urgencias fue de una hora en 19 pacientes $(31 \%)$, seguido por un tiempo menor de una hora en $16(27 \%)$. Diez pacientes arribaron a las 4 horas (17\%) y siete (12\%) a las dos horas de ocurrido el evento que originó la fractura. El diagnóstico más frecuente en el estudio fue fractura diafisiaria cerrada de tibia en 26 casos (44\%), seguido por el de fractura cerrada de fémur en 18 (30\%), fractura abierta de tibia en 10 (17\%), 
fractura abierta de fémur en $3(5 \%)$, fractura tibial bilateral en un caso $(1.7 \%)$ y finalmente fractura bilateral de fémur en otro (1.7\%). El 17\% de los pacientes presentaron dos o más fracturas de huesos largos o fractura concomitante de pelvis (10 casos) y el $83 \%$ cursaron con fractura aislada del fémur o tibia (49 casos).

A tres pacientes se les diagnosticó politraumatismo al ingreso a urgencias (5\%), mientras que el $95 \%$ restante (56 casos) mostraron compromiso sólo del sistema musculoesquelético. El trauma de alta energía causó $81 \%$ de las fracturas diafisiarias de fémur y/o tibia (48 casos) y solo el 19\% fueron consecuencia de trauma de baja energía (11 casos).

Nueve pacientes de los 68 (13\%) que tuvieron diagnóstico de fractura diafisiaria de fémury/otibia fueron excluidos del estudio por no seguir el protocolo de líquidos endovenosos y oxígeno en urgencias. Se tomaron gases arteriales al ingreso al $83 \%$ de los pacientes (49 casos). Al 56\% (33 casos) se les realizó la estabilización quirúrgica en las primeras 24 horas de su ingreso, al $10 \%$ entre 24 y 48 horas (6) al 19\% (11) entre las 48 y 72 horas y al 15\% (9) después de 72 horas.

Fueron estabilizados de manera inicial con clavos endomedulares fresados 32 pacientes (54\%) y el tiempo quirúrgico promedio utilizado en la osteosíntesis fue de 122 minutos. Mediante fijadores externos 22, cirugía en la cual el promedio fue de 88 minutos. En cuatro se emplearon placas con una duración de 110 minutos y por último un paciente con un clavo endomedular no fresado en 120 minutos. El tiempo quirúrgico promedio utilizado en los enlclavijamientos endomedulares fresados bloqueados de tibia fue de 109 minutos y en fémur 146 minutos.

En la mayoría de los casos que al comienzo fueron estabilizados con fijador externo, se realizó la osteosíntesis definitiva con clavos endomedulares fresados (11 casos, 50\%), seguido por placas (18\%, 4 casos). El tiempo quirúrgico promedio utilizado en la estabilización quirúrgica de las fracturas de fémur y/o tibia fue de 110 minutos. El 53\% (32 casos) de los pacientes se estabilizaron en un lapso que varió entre 40 y 99 minutos, el $32 \%$ (19 casos) entre 100 y 149 minutos, el $12 \%$ ( 7 casos). Por último un paciente requirió más de 200 minutos para la estabilización quirúrgica de sus fracturas. El promedio de estadía en el pabellón de los pacientes incluidos en el estudio fue de 6.4 días y en la UCI de 6 días.

Dos pacientes del estudio presentaron SEG (3.4\%), uno con fractura aislada de fémur y otro en el grupo de los polifracturados. El promedio de edad fue de 28 años y ambos del sexo masculino. El tiempo transcurrido entre el trauma y el ingreso fue menor de una hora en los dos casos. Ambos presentaron fractura diafisiaria cerrada de fémur y el polifracturado cursó con fractura de tibia contralateral abierta. Ninguno tenía diagnóstico de politraumatismo aunque fueron traumas en que la energía implicada fue alta.

A los dos pacientes se les realizó toma de gases arteriales a su ingreso, observando hipoxia ( $\mathrm{paO} 2$ menor de $60 \mathrm{~mm}$ de $\mathrm{Hg}$ ) en uno de ellos. Fueron estabilizados en las primeras dos horas con fijadores externos en un lapso quirúrgico de 100 (paciente con fractura de tibia contralateral) y de 60 minutos. El promedio de estadía en UCI de los dos pacientes fue cuatro días y en el pabellón de 12 días. Ninguno de los dos falleció.

Cabe anotar que dos de los nueve pacientes sin incluír en el estudio, por no haber aplicado el protocolo de líquidos endovenosos y oxígeno a su ingreso a urgencias, presentaron SEG. Ambos eran de sexo masculino con un promedio de edad de 24 años, cursaron con fracturas de fémur fueron traídos a la institución dentro de las primeras dos horas después de ocurrido el trauma y uno de ellos falleció por trauma cerrado de tórax (politraumatizado). El otro se estabilizó con un fijador externo a las 56 horas del trauma con un tiempo quirúrgico de 65 minutos. El paciente que murió por politraumatismo se estabilizó con fijadores externos en las tres primeras horas del trauma, con un tiempo quirúrgico de 120 minutos. El promedio de estadía en UCI fue de 2.5 días y en piso de 4.5 días.

\section{Discusión}

Las manifestaciones clínicas del SEG se conocen hace más de 100 años, luego de la descripción inicial de Zenker en 1861. Sin embargo, aun persiste el debate acerca de su diagnóstico y manejo clínico. ${ }^{10}$ Además, las mayores revisiones del SEG datan de hace más de 
20 años y los efectos de los cambios en el manejo de esta entidad, la estabilización temprana de las fracturas y su manejo en la UCI no se han publicado.

La incidencia varía en las distintas series entre 0.5 y $3 \%$ para fracturas aisladas de huesos $\operatorname{largos}^{4}$ y de 5 a $10 \%$ para pacientes polifracturados. ${ }^{8}$ En nuestro estudio fue de $3,4 \%$ para la totalidad de los pacientes, $2.08 \%$ para aquellos con fracturas asiladas de fémur y tibia, $\mathrm{y}$ en los polifracturados fue del $10 \%$. Es decir, el resultado en nuestro estudio es similar al de la literatura.

El estudio realizado por Bulger, Smith, Maier y Jurkovich $^{6}$ durante diez años revela los datos de 3026 pacientes con fracturas de huesos largos, encontrando una incidencia de 0,9\% del SEG, predominio del sexo masculino y una edad promedio de 31 años. En nuestro estudio fueron en su mayoría del sexo masculino (88\%) y el rango de edad más frecuente entre los 25 y 35 años.

La literatura sugiere una alta frecuencia en pacientes con más de una fractura de huesos $\operatorname{largos},{ }^{8}$ pero no reporta una predisposición a que se presente con fractura del fémur o la tibia. Nuestro estudio coincide con estas publicaciones encontrando una incidencia del $10 \%$ en los pacientes polifracturados. Además, el diagnóstico más relacionado con el SEG fue fractura diafisiaria cerrada de fémur. Ninguno de los pacientes con politraumatismo cursó con SEG.

La fijación temprana de las fracturas se considera de gran importancia en la reducción de las complicaciones pulmonares, incluyendo el SEG. Sin embargo, algunos autores afirman que la fijación con clavos endomedulares puede aumentar el riesgo de complicaciones pulmonares. ${ }^{10}$ La mayoría de los pacientes de este estudio $(56 \%)$ se estabilizaron dentro de las primeras 24 horas; los que desarrollaron SEG se estabilizaron en las primeras dos horas de ocurrido el trauma y el tiempo quirúrgico usando fijadores externos fue de 80 minutos, similar al transcurrido en aquellos que no desarrollaron esta entidad.

El tipo de osteosíntesis inicial utilizada en los dos pacientes con SEG fue de fijadores externos, los cuales no invaden el canal medular y por tanto no predisponen al desarrollo de esta patología. La mortalidad por SEG está reportada en años recientes entre 5 y $10 \% .{ }^{10} \mathrm{En}$ nuestro estudio fue $0 \%$.
Con los resultados del presente análisis no se puede llegar a conclusiones válidas debido al escaso número de casos de SEG. Sin embargo, se observa que la edad, sexo, tiempo transcurrido entre el trauma y el ingreso a urgencias, tiempo entre el trauma y la estabilización quirúrgica de la fractura, diagnóstico, tiempo quirúrgico o tipo de osteosíntesis, no fueron factores claros y contundentes para el desarrollo del SEG. Esta observación coincide con la literatura. ${ }^{10} \mathrm{El}$ riesgo de desarrollar esta entidad depende de las respuestas biológicas selectivas del huésped, como la inflamatoria a los ácidos grasos, más que del origen de los émbolos grasos. ${ }^{10}$

Por último, cabe anotar que en el trabajo realizado por Navarro y Bayona en el año 2003 en el hospital de San José, ${ }^{11}$ se estudió el manejo en urgencias de los pacientes con fracturas diafisiarias de fémur y/o tibia, hallando que el 79\% no recibía adecuados líquidos endovenosos y el $81 \%$ no tenía manejo con oxígeno. En este trabajo la incidencia del SEG fue de 7\%, mayor que la reportada en la literatura.

\section{Referencias}

1. Bucwalter, J A, Einhorn, TA. Simon S R. Orthopaedic basic science: Biology an biomechanics of the musculoesqueletal system. 2 nd edition Rosemont Illinois, 2000.

2. Manning J B Bach A W Herman C M Carrico C J Fat release after femur nailing in the dog. J. Trauma 1983; 23: 322-26.

3. Peltier LF OFat embolism: A perspective. Clin Orthop 1988; 232:263-70.

4. Gossling P. Fat embolism syndrome. A Review of the Pathophisiology and physiology basis of treatment Clin Orthop. 1982; 165: 68-82.

5. Capan, M. Monitoring for suspected pulmonary embolism. Anesthesia Clinics of 1993 p.691-96.

6. Modig J Busch C Olerud S. Saldeen T Pulmonary microembolism during intramedullary orthopaedic trauma. Acta Anaesth Scand 1974; 18: 133-43.

7. Peltier C Evarts: Fat embolism. Arch Surg 1974; 109:12-16.

8. Riska E B, Myllynen P Fat embolism in patients with multiple injuries. J. Trauma1982; 22: 891-94.

9. Browne J Trafton G Swiontkoswsky. Skeletal Trauma. 2 nd edition, Rosemont Illinois, Editores (WB Saunders Company), 1998; p. 443-46.

10. Bulger S M Jurkovich: Fat embolism syndrome: A 10 - year review. Arch Surg 1997; 132 (4): 435-39.

11. Navarro B. Sindrome de embolismo graso en fracturas diafisiarias de fémur y tibia en el Hospital de San José Bogotá: Hospital de San José: 1998, p.30. 